\title{
Electrochemical Analysis of Ultrathin Polythiolsiloxane Films for Surface Biomodification
}

\author{
Hao-Chun Chiang $\mathbb{D}$ and Rastislav Levicky \\ Chemical and Biomolecular Engineering, NYU Tandon School of Engineering, Brooklyn, NY 11201, USA \\ Correspondence should be addressed to Hao-Chun Chiang; hcc293@nyu.edu
}

Received 13 July 2018; Revised 8 September 2018; Accepted 16 September 2018; Published 3 October 2018

Academic Editor: Shengshui Hu

Copyright (C) 2018 Hao-Chun Chiang and Rastislav Levicky. This is an open access article distributed under the Creative Commons Attribution License, which permits unrestricted use, distribution, and reproduction in any medium, provided the original work is properly cited.

\begin{abstract}
The ability of different crosslinkers to crosslink nanometer thick films of the polymer poly(mercaptopropyl)methylsiloxane (PMPMS), thus stabilizing these films on solid supports, was investigated. The four crosslinkers included 1,11-bismaleimidotriethyleneglycol $\left(\mathrm{BM}(\mathrm{PEG})_{3}\right)$, tris-(2-maleimidoethyl)amine (TMEA), bismaleimidohexane (BMH), and 1,1' ${ }^{\prime}$-(methylenedi-4,1phenylene) bismaleimide (BMDPM). PMPMS films treated with the four crosslinkers were compared in the effectiveness of achieved crosslinking, continuity and stability of the films to rearrangement at elevated temperatures, and modification with singlestranded DNA. The results of electrochemical analyses show that more hydrophilic crosslinkers had difficulty reacting fully with PMPMS thiols, even in these nanometer thin layers. This observation highlights the critical importance of selecting crosslinkers that are chemically compatible. Optimal selection of crosslinker yielded films in which the polymer film was largely incapable of rearranging, even at elevated temperatures, yielding reproducible and stable layers. These results validate use of these supports for applications such as monitoring thermal denaturation of immobilized DNA duplexes.
\end{abstract}

\section{Introduction}

Thermal stability of biomolecular films on solid supports is key to their diagnostic and related applications which typically require elevated temperatures for optimal performance, as well as to basic research on such layers. For example, thermal denaturation has been used to analyze thermodynamics of hybridization on solid supports including effects of chain length [1-3], probe associations $[4,5]$ and surface density [6], probe-surface interactions $[7,8]$, salt concentration [9-14], and how such systems perform in commercial technologies [15]. Similar needs arise for immobilized protein films such as antibodies or enzymes [16-18], where the impact of the surface environment on stability or activity of the protein could be correlated with determination of its denaturation transition and unfolding. Such applications require immobilization chemistries capable of performing at temperatures approaching physical limits, like the boiling point of water. For instance, studies of DNA monolayers that require determination of melting curves expose such films for hours to temperatures of up to $80^{\circ} \mathrm{C}$ [14].
On glass type supports, organosilane chemistry provides stable immobilization [14]. Other common supports include conductive materials such as gold, which are especially popular in academic research but are also encountered in commercial systems [19]. Gold provides convenient immobilization via thiol moieties, tailorable surface chemistry, stability under aqueous buffers, and convenient interface for electrochemical characterization. Often, biomolecules are immobilized to gold using a single thiolate bond; this approach, however, is susceptible to degradation by time and temperature. Approaches based on multivalent attachment through several thiolate bonds have produced significantly more stable immobilization [20-24]. Fragoso and O'Sullivan also reported a markedly more robust attachment based on reduction of individual diazonium moieties and showed it to be significantly more stable than even dithiol attachment [25]. Another class of methods utilize polymeric base layers that are a few nanometers thick and adhere irreversibly to gold, to which the biomolecules are conjugated through stable covalent bonds [26]. One such very effective approach is to use polythiolsiloxane base films in which the polymers 
are bound to the gold support through multiple thiolate bonds, with remnant thiols serving for attachment of the biomolecules, typically through thioether linkages $[27,28]$.

Due to the typical application of these systems in bioelectronic sensors, their electrochemical characterization is of great interest. In this paper, electrochemical methods are applied to evaluate stability-related properties of polythiolsiloxane films as a function of crosslinker selection, where the crosslinker serves to simultaneously bind the base layer chains to each other as well as to activate the layer for attachment of biomolecules. Four commercially available, maleimide-based crosslinkers are evaluated for their ability to realize high degrees of crosslinking of nanometer thick layers of the polythiolsiloxane polymer poly(mercaptopropyl)methylsiloxane (PMPMS), with the further goal to maximize tolerance to high temperatures. Realization of optimal stability requires crosslinkers to partition into the polymer layer so as to gain access to its thiol groups, a consideration that turns out to be crucial even for these ultrathin films. The impact of crosslinking on hydrophobicity and other properties of the crosslinked support are also considered. The crosslinker most effective for creating stable layers is used to demonstrate measurement of melting transitions in immobilized DNA films [4].

\section{Materials and Methods}

2.1. Materials. Poly(mercaptopropyl)methylsiloxane (PMPMS, average degree of polymerization of $40,95 \%$, Gelest Inc), $N$-hydroxysuccinimide ester of ferrocene carboxylic acid (FcCA-NHS, 98 \%, Tokyo Chemical Industry Co. Ltd.), 1,11bismaleimidotriethyleneglycol $\left(\mathrm{BM}(\mathrm{PEG})_{3}, 98 \%\right.$, Thermo Fisher Scientific Inc.), tris-(2-maleimidoethyl)amine (TMEA, 98\%, Thermo Fisher Scientific Inc.), bismaleimidohexane (BMH, 95\%, Sigma-Aldrich), $N, N^{\prime}$-(4,4'-diphenylmethane) bismaleimide (BMDPM, $95 \%$, Sigma-Aldrich), potassium ferricyanide $\left(\mathrm{K}_{3} \mathrm{Fe}(\mathrm{CN})_{6},>99.0 \%\right.$, Sigma-Aldrich), and 6-(ferrocenyl)hexanethiol (FSH, 97\%, Sigma-Aldrich) were used as received. Table 1 provides the molecular weight and arm lengths for the four crosslinkers tested, and the chemical structures are shown in Figure 1. Aqueous buffers were prepared from deionized water $(18.2 \mathrm{M} \Omega \mathrm{cm})$. Sodium phosphate buffer (SPB), consisting of $0.5 \mathrm{~mol} \mathrm{~L}^{-1}$ phosphate groups, $\mathrm{pH}$ 7.0, was used for all hybridization measurements. DNA oligodeoxyribonucleotides were purchased from Integrated DNA Technologies (IDT) and were purified by reverse phase high-performance liquid chromatography by the manufacturer. $5^{\prime}$ Disulfide-terminated DNA 20 mer "probes" were used for immobilization and had the sequence $5^{\prime} \mathrm{HO}-\left(\mathrm{CH}_{2}\right)_{6}$-S-S- $\left(\mathrm{CH}_{2}\right)_{6}$-AGG AAG AGA AAG AAG GAG AA- $\left.\mathrm{CH}_{2}-\mathrm{CH}(\mathrm{OH})-\mathrm{CH}_{2}-\mathrm{O}-\left(\mathrm{CH}_{2}\right)_{3}-\mathrm{NH}_{2}\right) 3^{\prime}$. The complementary "target" sequence used for hybridization was 5 " TTC TCC TTC TTT CTC TTC CT - $\mathrm{CH}_{2}-\mathrm{CH}(\mathrm{OH})-\mathrm{CH}_{2}-\mathrm{O}-$ $\left.\left(\mathrm{CH}_{2}\right)_{3}-\mathrm{NH}_{2}\right) 3^{\prime}$. The $3^{\prime}$ propylamine group on both the probe and target strands was used for labeling with the electroactive FcCA tags.

2.2. Preparation of PMPMS Layers. The preparation of gold disk working electrodes was described previously [4].
Electrodes were polished manually and electrochemically, followed by measurement of their roughness factor $r$ from differential capacitance under $1 \mathrm{mM} \operatorname{NaF}[29,30]$. Next, the cleaned electrodes were washed with deionized water, rinsed with dimethyl formamide (DMF), and transferred, while still wet, into $10 \mathrm{mM}$ solutions (by monomer residue) of PMPMS in DMF while stirring for $2 \mathrm{hrs}$. After chemisorption of PMPMS, electrodes were rinsed extensively with DMF to remove unattached PMPMS. The PMPMS-coated and washed electrodes were next immersed in $1 \mathrm{mM}$ solutions of one of the four crosslinkers in DMF, while stirring, for durations of up to $17 \mathrm{hrs}$, followed by thorough rinsing with DMF and drying under compressed nitrogen. This step crosslinks the separate PMPMS chains into an infinite network, provided crosslinkers react so as to span between thiols from different PMPMS chains. Maleimides that are left unreacted can be used for subsequent immobilization of thiol-bearing biomolecules.

2.3. Preparation of DNA Monolayers. PMPMS films, crosslinked and maleimide-activated as described above, were immediately placed in $1 \mu \mathrm{M}$ solutions of thiol-terminated DNA probes in SPB at room temperature for 1 hour. Thiolterminated probes were prepared by cleaving the $5^{\prime}$ disulfide with dithiothreitol, followed by purification and resuspension in SPB as previously described [4]. Control experiments used the as-received, disulfide-protected probe DNA. After probe immobilization, electrodes were rinsed with deionized water and SPB.

2.4. Measurement of PMPMS Thickness. The thickness of PMPMS films was estimated from the differential capacitance per area, $C_{d}$, measured with electrochemical impedance spectroscopy (EIS). Previously, using PMPMS films prepared on gold-coated slide supports, this approach was shown to yield thickness values in good agreement with ellipsometric measurements [28]. EIS measurements used $3 \mathrm{mV}$ (rms) amplitude AC modulation swept from $10 \mathrm{~Hz}$ to $10 \mathrm{kHz}$, on top of a DC offset of $0 \mathrm{mV}$ versus an $\mathrm{Ag} / \mathrm{AgCl} / 3 \mathrm{M} \mathrm{NaCl}$ reference. A Pt wire counterelectrode was used on a $\mathrm{CH}$ Instruments workstation. The experimental impedance $Z$ was interpreted in terms of an equivalent circuit composed of a solution resistance $R_{s}$ in series with a constant phase element (CPE) representing the charging response of the electrode. A CPE was used instead of a pure capacitor to allow for dispersion in the capacitive charging caused by roughness or chemical heterogeneity of the interface $[31,32]$. The impedance of a $\mathrm{CPE}$ is given by

$$
Z_{C P E}=\frac{1}{\left((j \omega)^{n} Q\right)}
$$

where $\omega$ is the frequency, $n(0 \leq n \leq 1)$ and $Q$ are parameters characterizing the CPE and $j$ is the square root of -1 . An $n$ of 1 corresponds to an ideal capacitor, while $n=0$ would be a resistor with resistance $1 / Q$. An effective capacitance per area, $C_{d}$, is calculated from [32]

$$
C_{d}=\frac{\left(R_{S}^{1-n} Q\right)^{1 / n}}{A}
$$




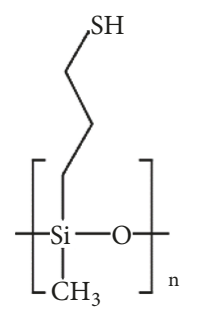

PMPMS<smiles>O=C1C=CC(=O)N1CCOCCOCCOCCN1C(=O)C=CC1=O</smiles>

$\mathrm{BM}(\mathrm{PEG})_{3}$<smiles>O=C1C=CC(=O)N1CCN(CCN1C(=O)C=CC1=O)CCN1C(=O)C=CC1=O</smiles>

TMEA<smiles>O=C1C=CC(=O)N1CCCCCCN1C(=O)C=CC1=O</smiles>

BMH<smiles>O=C1C=CC(=O)N1c1ccc(Cc2ccc(N3C(=O)C=CC3=O)cc2)cc1</smiles>

BMDPM

FIGURE 1: Chemical structures of the polythiolsiloxane polymer PMPMS and the crosslinkers tested.

TABLE 1: Crosslinker properties. "Arm length" refers to distance between the two maleimide C=C bonds. Arm lengths for BMH and BMDPM are predictions from Scifinder, while those for $\mathrm{BM}(\mathrm{PEG})_{3}$ and TMEA were provided by the manufacturer.

\begin{tabular}{lccc}
\hline Name & Abbreviation & MW g/mol & Arm length $\AA$ \\
\hline 1,11-Bismaleimidotriethyleneglycol & BM(PEG) & 352 & 17.3 \\
Tris-(2-maleimidoethyl)amine & TMEA & 386 & 10.3 \\
Bismaleimidohexane & BMH & 276 & 13.0 \\
$1,1^{\prime}$-(methylenedi-4,1-phenylene) Bismaleimide & BMDPM & 358 & 15.0 \\
\hline
\end{tabular}

where $A$ is the actual electrode area given by the product of the geometric area and the roughness factor $r . R_{s}, \mathrm{Q}$, and $n$ were determined by fitting of the experimental frequencydependent impedance to a model consisting of a CPE element in series with the solution resistance; $C_{d}$ was then calculated from (2). Fitted values of $n$ for neat PMPMS films were 0.98 or higher, indicating that the PMPMS films exhibited close to ideal capacitor behaviour. Based on this observation, the PMPMS thickness was calculated from the parallel plate capacitor result

$$
C_{d}=\frac{\varepsilon \varepsilon_{o}}{d}
$$

where $\varepsilon=6.0$ is the dielectric constant of PMPMS [33], $\varepsilon_{o}$ is the permittivity of free space, and $d$ is the PMPMS layer thickness. To minimize contribution of the diffuse double layer to $C_{d}$ and hence $d$, EIS measurements were performed in a high ionic strength, $1 \mathrm{M} \mathrm{SPB}$, buffer. Under these conditions $C_{d}$ is dominated by capacitance of the PMPMS layer and provides thickness values in good agreement with ellipsometric measurements [28].

Figure 2 shows examples of EIS data in the form of a Nyquist plot of the measured imaginary $Z^{\prime \prime}$ and real $Z^{\prime}$ impedance components, as a function of crosslinking time for PMPMS layers crosslinked with BMDPM. Addition of crosslinker caused a clear change from the uncrosslinked layer at $0 \mathrm{~min}$. Fits for the 0 and $30 \mathrm{~min}$ datasets are also plotted (solid lines). For the assumed model of a series $R_{S}$ and CPE circuit, the experimental impedances are fit using $Z^{\prime}=R_{S}+\cos (\pi n / 2) /\left(Q \omega^{n}\right)$ and $Z^{\prime \prime}=-\sin (\pi n / 2) /\left(Q \omega^{n}\right)$ by varying $R_{S}, Q$, and $n$. The effective capacitance $C_{d}$ then follows from (2).

2.5. Measurements of PMPMS Continuity. The physical continuity of PMPMS layers was investigated by monitoring electron transfer across the layer from ferri/ferrocyanide $\left[\mathrm{Fe}(\mathrm{CN})_{6}\right]^{3-/ 4-}$ anions in solution. Continuous films prevent the ions from approaching the gold surface, diminishing their redox activity [34]. These experiments used test solutions of $10 \mathrm{mM}$ potassium ferri/ferrocyanide $\left[\mathrm{Fe}(\mathrm{CN})_{6}\right]^{3-/ 4-}$ redox couple in $200 \mathrm{mM} \mathrm{KCl}$. An $\mathrm{AgCl}$ coated silver wire served as a quasi-reference electrode, with a Pt wire counterelectrode. Cyclic voltammograms (CVs) were measured from -300 to $600 \mathrm{mV}$ with a scan rate of $20 \mathrm{~V} / \mathrm{s}$. The redox potential of the $\mathrm{Fe}(\mathrm{CN})_{6}{ }^{3-/ 4-}$ redox couple was observed at $250-300 \mathrm{mV}$. All measurements were initiated with fresh test solutions to minimize possible degradation of the redox couple. 
2.6. Melting Curve Measurements. Melting curve measurements on immobilized duplexes were performed as in prior work [4]. In brief, target oligonucleotides were labeled with ferrocene FcCA-NHS tags, followed by purification using reversed-phase HPLC. The FcCA-labeled targets were hybridized to PMPMS-anchored DNA monolayers using 25 nM solutions in $0.5 \mathrm{M} \mathrm{SPB}$ and the extent of hybridization was tracked as a function of temperature. Hybridization measurements were performed under a nitrogen purge to minimize dissolved oxygen, which contributes to ferrocene degradation via the oxidized ferricinium state [35]. A temperature controlled, jacketed electrochemical cell (Gamry Instrument, Warminster, PA) was used. Experiments started at a high temperature of $75^{\circ} \mathrm{C}$, were cooled at 0.3 degrees $/ \mathrm{min}$ to $30^{\circ} \mathrm{C}$, and then heated back to $75^{\circ} \mathrm{C}$. CV curves were measured as a function of temperature during both the cooling and heating cycles, and the extent of hybridization was determined by integrating the ferrocene faradaic peak as described below. All CV measurements used a potential sweep rate of $20 \mathrm{~V} \mathrm{~s}^{-1}$, applied between 0 and $700 \mathrm{mV}$ to capture the ferrocene peak.

2.7. Calculation of DNA Surface Coverage. DNA coverage, in molecules/area, was derived from the charge $Q$ needed to switch the FcCA tags' oxidation state. $Q$ was determined from cyclic voltammetry traces (range: 0 to $700 \mathrm{mV}$, scan rate: 20 $\mathrm{V} / \mathrm{s}$ ) by integrating the area of the ferrocene tag oxidation peak after correction for baseline current and converting the potential axis to time [36]. Since each strand has just one label, the corresponding molecular coverage, $S$, can be calculated using

$$
S=\frac{Q}{\left(e A_{g} r\right)}
$$

Here, $e=1.60 \times 10^{-19}, \mathrm{C}$ is the elementary charge, $A_{g}=$ $0.02 \mathrm{~cm}^{2}$ is the geometric electrode area, and $r$ is the surface roughness factor.

\section{Results and Discussion}

The extent of crosslinking was expected to be a primary parameter governing stability of the base polymer layer; moreover, by deactivating PMPMS thiols it serves to prevent their subsequent participation in immobilization of thiolcarrying biomolecules through alternate pathways such as formation of disulfide bridges [37]. Following this reasoning, crosslinkers were first ranked according to their ability to reach high extents of conversion of PMPMS thiols. The most promising crosslinkers were then investigated further with respect to optimization of stability and electrochemical properties of the films.

3.1. Crosslinker Effectiveness. The four crosslinkers were evaluated for their ability to maximize crosslinking. Crosslinked PMPMS films, prepared as described earlier, were exposed to solutions of disulfide-terminated probes. While the disulfide groups would be inert toward any unreacted crosslinker maleimides in the films, they could undergo exchange reactions with remnant PMPMS thiols. In the desired case all

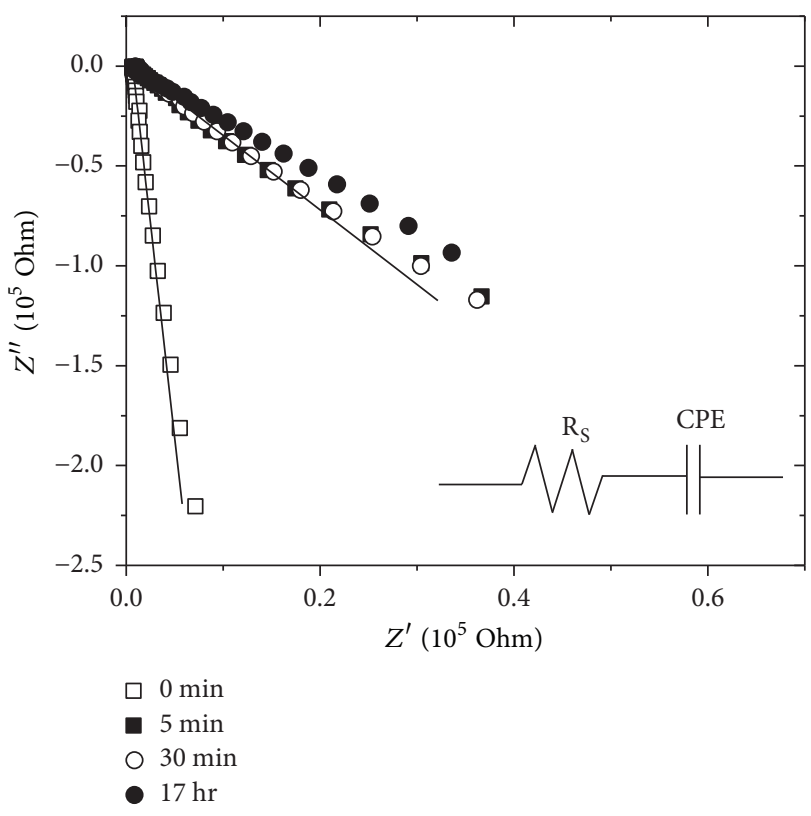

FIGURE 2: EIS data for a PMPMS layer crosslinked with BMDPM for the indicated time. Calculated fits, using an equivalent circuit of a solution resistance in series with a constant phase element, are shown for the 0 and $30 \mathrm{~min}$ data series as solid lines.

PMPMS thiols would be already consumed by crosslinker, so that there should be no immobilization of the probes.

The assays were performed by immersing crosslinked PMPMS films in $1 \mathrm{nM}$ solutions of $5^{\prime}$ disulfide-terminated probes in $1 \mathrm{M}, \mathrm{pH} 7.0 \mathrm{SPB}$ for $1 \mathrm{hr}$. Subsequent to probe immobilization, the layers were hybridized with complementary, FcCA-labeled targets present at $25 \mathrm{nM}$ in $0.5 \mathrm{M}, \mathrm{pH}$ $7 \mathrm{SPB}$, also for $1 \mathrm{~h}$. Presence of hybridization, as evidenced by FcCA electroactivity in $\mathrm{CV}$ traces, was thus indicative of probe immobilization and hence incomplete crosslinking of PMPMS.

As shown in Figure 3, PMPMS layers crosslinked with BMDPM and BMH did not exhibit FcCA activity, indicating that probes did not immobilize. This is consistent with achievement of high degrees of crosslinking, leaving few unreacted PMPMS thiols. In contrast, layers crosslinked with TMEA and $\mathrm{BM}(\mathrm{PEG})_{3}$ both exhibited FcCA peaks. This indicates that probes were able to immobilize through remnant PMPMS thiols due to incomplete crosslinking. The difference in crosslinking effectiveness between TMEA and $\mathrm{BM}(\mathrm{PEG})_{3}$ on one hand and $\mathrm{BMH}$ and BMDPM on the other likely reflects the more hydrophilic nature of the former two, Figure 1 . The more hydrophilic TMEA and BM(PEG) crosslinkers likely experience greater difficulty diffusing into the hydrophobic interior of the PMPMS layer and therefore are not able to access buried PMPMS thiols despite the layer being only $2 \mathrm{~nm}$ thick (cf. Figure 5). Since these crosslinkers were less effective, subsequent experiments focused on the relative strengths of the $\mathrm{BMH}$ and BMDPM crosslinkers.

3.2. Thermal Stability of Crosslinked PMPMS. Next, the stability of BMH and BMDPM-crosslinked films was compared 


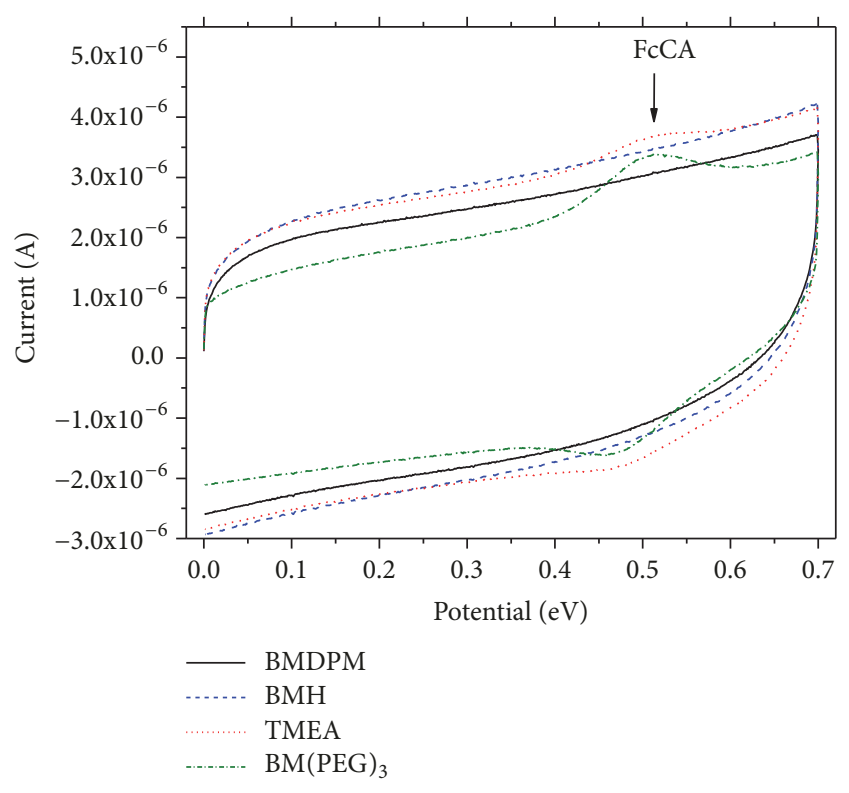

FIGURE 3: PMPMS thiols remaining after crosslinking support immobilization of disulfide-terminated probes through thiol/disulfide exchange reactions, leading to hybridization signal (peak at around $0.5 \mathrm{~V}$ ) for TMEA and $\mathrm{BM}(\mathrm{PEG})_{3}$ crosslinked films. Lack of signal in $\mathrm{BMH}$ and BMDPM treated films indicates near complete conversion of PMPMS thiols. Conditions of PMPMS deposition: 10 mM PMPMS in DMF, 2 hrs. PMPMS crosslinking: $1 \mathrm{mM}$ crosslinker in DMF, 17 hrs.

by monitoring the evolution of cyclic voltammetry (CV) curves after exposure of the films to $80^{\circ} \mathrm{C}$ for various durations. Measurements were performed in buffered $0.2 \mathrm{M} \mathrm{KCl}$ in the presence of $\left[\mathrm{Fe}(\mathrm{CN})_{6}\right]^{3-/ 4-}$ redox couple. Previously, XPS measurements in combination with EIS analysis revealed that PMPMS films on gold are continuous above $0.8 \mathrm{~nm}$ [33]. As the current films were nearly twice as thick (cf. Figure 5), they start as continuous and provided they remain so after the thermal treatment, without desorption or dewetting, the CV traces should remain unaltered. Such behaviour is expected if the PMPMS chains are extensively crosslinked to each other so that it is not possible for them to rearrange. On the other hand, rearrangement of the layer structure due to desorption or dewetting of PMPMS would lead to changes in film thickness and hence surface capacitance. Such changes can be monitored simply from the CV baseline current $I_{B}$, since $I_{B}$ is related to capacitance as $I_{B}=C_{d} v$ where $v$ is the scan rate. Should the PMPMS layer become extensively damaged with formation of holes then a pronounced increase in ferrocyanide $\left[\mathrm{Fe}(\mathrm{CN})_{6}\right]^{3-/ 4-}$ electroactivity would also be expected.

As shown in Figure 4(a), PMPMS films crosslinked with $\mathrm{BMH}$ rearranged after just a $15 \mathrm{~min}$ exposure to $80^{\circ} \mathrm{C}$, as primarily reflected in the increase in baseline currents. Longer exposures resulted also in observation of $\left[\mathrm{Fe}(\mathrm{CN})_{6}\right]^{3-/ 4-}$ redox activity. The broad peak widths and large separation between the $\left[\mathrm{Fe}(\mathrm{CN})_{6}\right]^{3-/ 4-}$ cathodic and anodic peaks are suggestive of heterogeneity in film thickness as well as electron transfer limitations being observed at the relatively fast
$20 \mathrm{~V} / \mathrm{s}$ scan rates. In contrast, films crosslinked with BMDPM remained minimally affected even after over an hour at $80^{\circ} \mathrm{C}$, Figure 4(b). This indicates that the BMDPM-crosslinked layer continued to fully cover the electrode surface and was not thinned or significantly damaged by heating, and did not form pinholes that would allow $\left[\mathrm{Fe}(\mathrm{CN})_{6}\right]^{3-/ 4-}$ to approach the electrode. For comparison, a control measurement on an uncrosslinked PMPMS layer is shown in Figure 4(c).

The amount of linker incorporation for both BMDPM and $\mathrm{BMH}$ were next investigated by monitoring the change in film thickness with time of exposure to the crosslinking solution, Figure 5. PMPMS films were dipped in the crosslinker solution for a designated time and then transferred to an aqueous buffer for determination of thickness as described in the experimental section. As seen in the figure, incorporation of both crosslinkers was complete within $5 \mathrm{~min}$, with no significant change with times of up to $17 \mathrm{hrs}$. Starting from uncrosslinked thickness of about $1.4 \mathrm{~nm}$, incorporation of BMDPM added about $1.0 \mathrm{~nm}$ while BMH caused an increase of around $0.5 \mathrm{~nm}$.

Given that linker incorporation required just a few minutes, the question arises whether it is necessary to wait as long as 17 hours for crosslinking to fully develop; in other words, whether incorporated linkers react right away with a second PMPMS thiol to form a crosslink, or whether the longer time is required. Uncrosslinked PMPMS is a polymer melt, but crosslinking will render it elastic. Previously, in CV experiments using crosslinked PMPMS at various temperatures it was found that the baseline current systematically and reversibly changed with temperature [28], an observation that was attributed to changing roughness of the PMPMS film due to temperature dependence of its interfacial tension. Higher extents of crosslinking should suppress such rearrangements due to greater constraints on mobility of the PMPMS chains; this suggests that it should be possible to qualitatively track the extent of film crosslinking from the difference in its baseline current between two temperatures. Accordingly, kinetics of BMDPM crosslinking were monitored by considering the difference in baseline current between $25^{\circ} \mathrm{C}$ and $50^{\circ} \mathrm{C}$ measured in 0.5 M SPB. As shown in Figure 6, longer crosslinking times reduced the temperature-induced change in baseline current, suggesting that the extent of crosslinking continued to increase over the investigated time of up to 17 hrs. The conclusion is that although crosslinker was largely incorporated within minutes, completing the formation of crosslinks required longer durations, on the order of hours.

An important parameter is the ratio of PMPMS thiols to crosslinker maleimides. This ratio determines whether sufficient maleimides are incorporated to react with all PMPMS thiols, as well as how many maleimides remain for attachment of biomolecules. From the $1.4 \mathrm{~nm}$ thickness of the film before crosslinking and a molecular volume of $0.23 \mathrm{~nm}^{3}$ per PMPMS residue, the number of PMPMS thiols within one $\mathrm{cm}^{2}$ area of the film was $(1.4 \mathrm{~nm})\left(1 \times 10^{14} \mathrm{~nm}^{2} / \mathrm{cm}^{2}\right) /\left(0.23 \mathrm{~nm}^{3}\right)=6.1$ $\times 10^{14}$ thiols $/ \mathrm{cm}^{2}$. Of this number, based on an earlier XPS analysis of Au-bound and unbound S 2p signals about 3.4 $\times 10^{14}$ thiols $/ \mathrm{cm}^{2}$ are expected to bind to the gold support [33], leaving about $(6.1-3.4) \times 10^{14}=2.7 \times 10^{14}$ thiols $/ \mathrm{cm}^{2}$ 


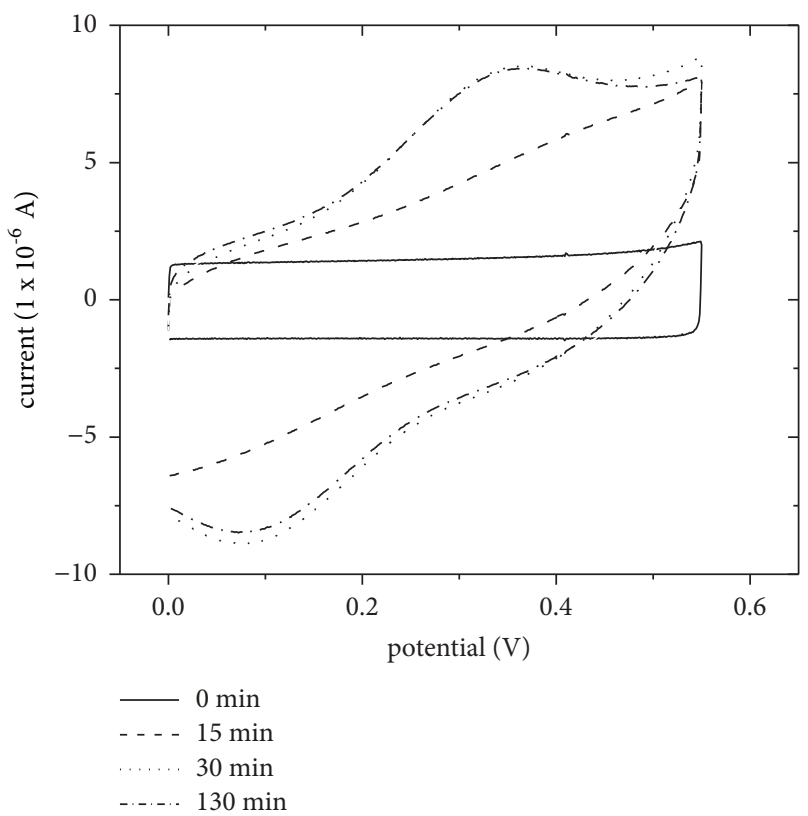

(a)

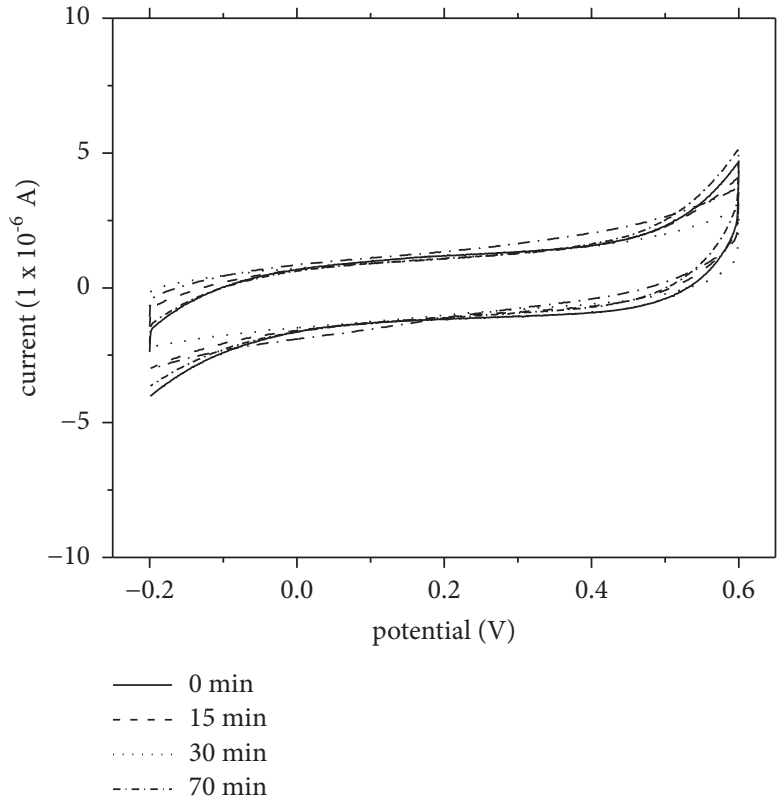

(b)

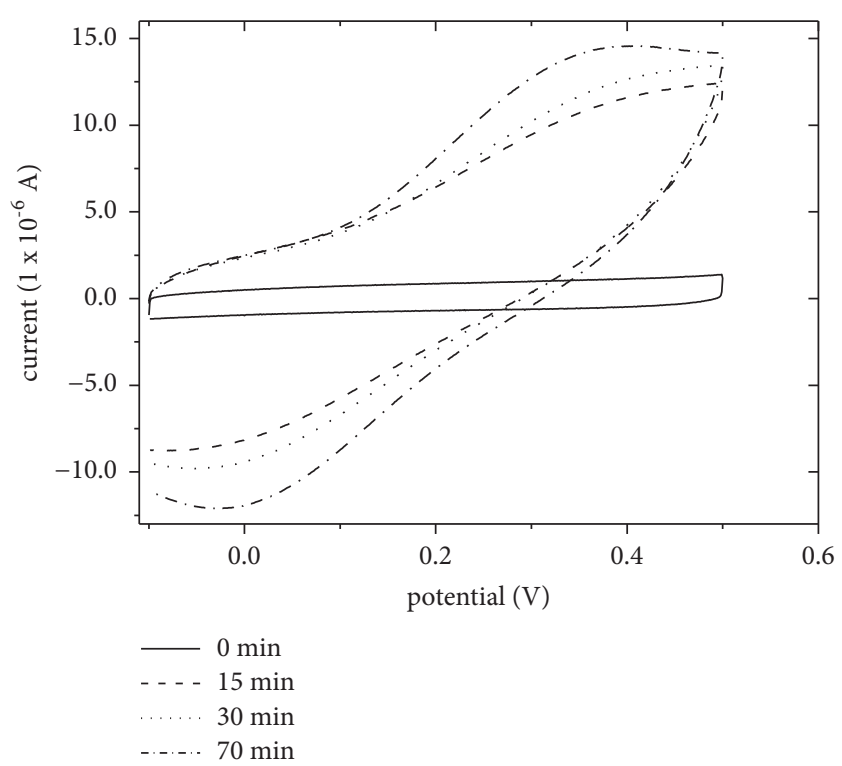

(c)

Figure 4: Cyclic voltammetry measurements on PMPMS layers crosslinked for 17 hours with $1 \mathrm{mM}$ (a) BMH and (b) BMDPM in DMF. The cured layers were immersed in $80^{\circ} \mathrm{C}$ deionized water for the indicated time, after which they were immersed in $\mathrm{K}_{3} \mathrm{Fe}(\mathrm{CN})_{6}$ solution for the CV measurements. PMPMS deposition: 10 mM PMPMS in DMF, 2 h. Data for an uncrosslinked PMPMS layer are shown in (c).

available for reacting with crosslinkers. From the change in film thickness and crosslinker molecular volumes (BMDPM: $0.41 \mathrm{~nm}^{3} /$ molecule; BMH: $0.35 \mathrm{~nm}^{3} /$ molecule), analogous calculations yield the amount of incorporated crosslinker as $2.5 \times 10^{14}$ molecules $/ \mathrm{cm}^{2}$ for BMDPM and $1.5 \times 10^{14}$ molecules $/ \mathrm{cm}^{2}$ for BMH. Since each crosslinker bears two maleimides, the maleimide to thiol ratio was $2\left(2.5 \times 10^{14}\right)$ to $2.7 \times 10^{14}$, or about 1.9:1 for BMDPM; analogous calculations yield 1.2:1 for BMH. These estimates support the conclusion that crosslinker maleimides were incorporated in excess and therefore sufficient to react with all available PMPMS thiols.
This is also consistent with the negligible attachment of DNA probes through formation of disulfide bridges, Figure 3. Uncertainties in these values may be significant, however, due to approximations including use of the dielectric constant for unmodified PMPMS to also calculate thicknesses of crosslinked films.

To confirm activation of the films for further modification, remnant maleimide activity after linker incorporation was measured. BMDPM-crosslinked (1 mM crosslinker, 17 hrs reaction time) PMPMS films were reacted with $1 \mathrm{mM} 6-$ (ferrocenyl) hexanethiol (FSH), and then the FSH coverage 


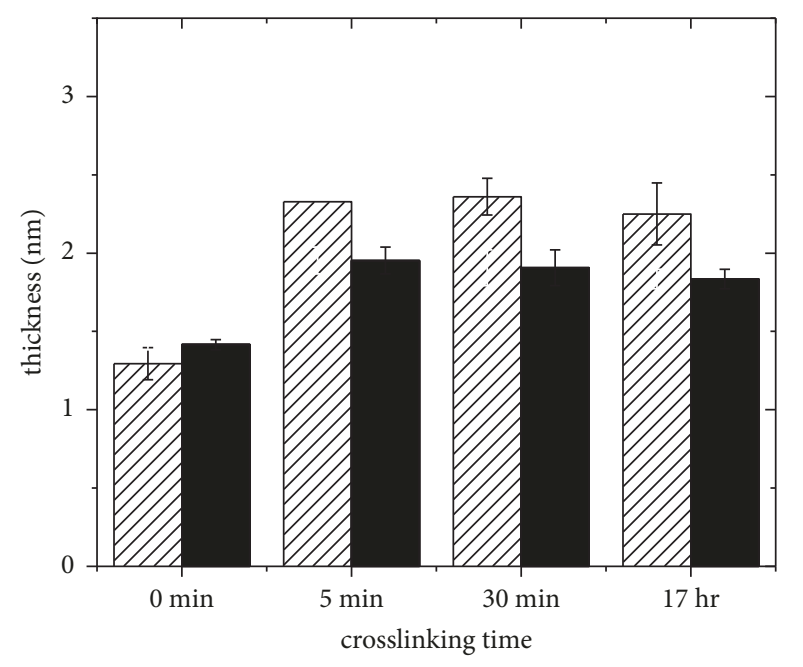

VIIIA BMDPM $\mathrm{BMH}$

FIGURE 5: Kinetics of linker incorporation as indicated by changes in monolayer thickness. The error bars correspond to standard deviation of two independent EIS measurements performed on the same sample.

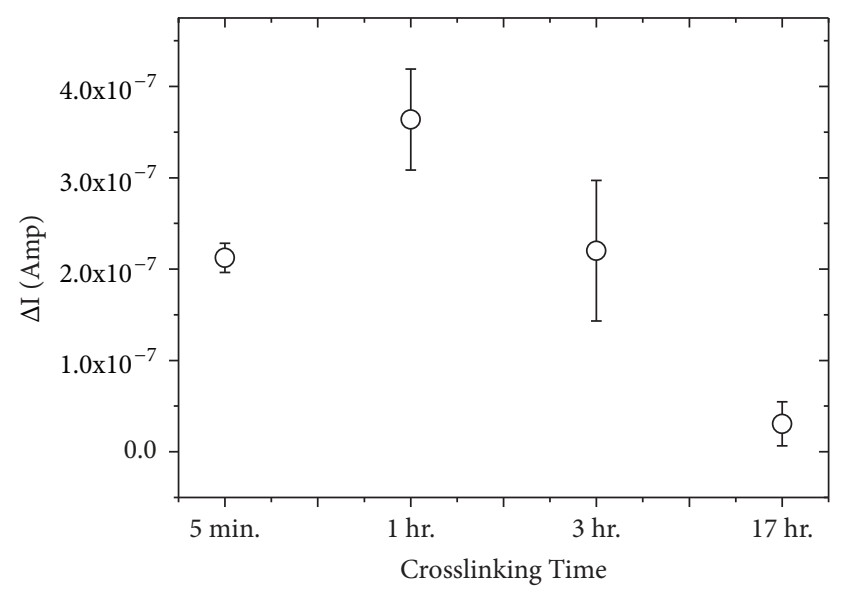

FIgURE 6: The difference in $\mathrm{CV}$ baseline current between two temperatures, $25^{\circ} \mathrm{C}$ and $50^{\circ} \mathrm{C}$, in $0.5 \mathrm{M} \mathrm{SPB}$, as a function of BMDPM crosslinking time. $\Delta I$ on the $y$-axis is the temperature-induced change in the baseline separation between forward and reverse $\mathrm{CV}$ sweeps, $\Delta I=\left(I_{\text {Forward }}-I_{\text {Reverse }}\right)_{25 \mathrm{C}}-\left(I_{\text {Forward }}-I_{\text {Reverse }}\right)_{50 \mathrm{C}}$, measured at a bias of $0.1 \mathrm{~V}$.

was quantified from integrating its CV peak, analogous to (4). These measurements yielded FSH immobilization coverage of $5 \times 10^{12}$ molecules $/ \mathrm{cm}^{2}$, indicating that at least this coverage of maleimide was available to support further modification with biomolecules. Moreover, taking the earlier estimate of $2.7 \times 10^{14}$ thiols $/ \mathrm{cm}^{2}$ available for crosslinking, with $5 \times$ $10^{12}$ thiols $/ \mathrm{cm}^{2}$ reacting with BMDPM crosslinker in a monodentate fashion and the rest assumed to form crosslinks, predicts an incorporated BMDPM coverage of about 1.3 $\times 10^{14}$ molecules $/ \mathrm{cm}^{2}$. This is about half the $2.5 \times 10^{14}$ molecules $/ \mathrm{cm}^{2}$ derived from film thickness measurements. The discrepancy could reflect a number of uncertainties in these estimates, including not correcting for decrease in PMPMS dielectric constant due to crosslinking, what would overestimate the film thickness and hence incorporation of crosslinker, as well as incomplete reaction of maleimides with FSH, e.g., due to inaccessibility of some maleimides and/or their hydrolysis to unreactive maleamic acid.

3.3. Melting Curve Measurements. Based on their excellent stability, BMDPM-crosslinked films were selected for demonstration of melting curve measurements on DNA films. Thiol-terminated DNA probes were immobilized to BMDPM-crosslinked PMPMS films, placed under a stirred solution of complementary FcCA-labeled target DNA present in excess, and the extent of hybridization was measured as a function of temperature. Melting curve measurements used a temperature scan rate of $0.3^{\circ} \mathrm{K}$ per minute. An example of a melting curve is shown in Figure 7.

The main feature of note in Figure 7 is the near superposition of heating and cooling curves. This superposition highlights the stability of these films over several hours at elevated temperatures of up to $348^{\circ} \mathrm{K}\left(75^{\circ} \mathrm{C}\right)$, since the same melting behaviour is observed for both the cooling and subsequent heating ramps. The superposition also indicates that the melting data were obtained under equilibrium conditions. The inset in Figure 7 shows a typical CV scan including the redox peaks of the FcCA-labeled DNA target. The data confirm that the DNA probes remained attached to the surface and that their hybridization activity was preserved during the heating. BMDPM-crosslinked PMPMS films are therefore well suited for applications such as study of hybridization thermodynamics on solid supports $[3,4]$. The melting temperature on the solid support can be estimated from the $50 \%$ hybridization state as $321^{\circ} \mathrm{K}$. This value can be compared to the solution prediction of $335^{\circ} \mathrm{K}$ calculated using UNAFOLD [38]. The lower temperature for melting of immobilized duplexes is not surprising, given that hybridization on solid supports tends to be less thermodynamically favorable than that in solution $[1,4,9,39]$.

\section{Conclusion}

This electrochemical study investigated how crosslinkers stabilize nanometer thick films of initially uncrosslinked polysiloxane polymers, which are used as base layers for attachment of biomolecules to gold. Several conclusions from this system are expected to be of general interest. First, it is essential to select crosslinkers compatible with the polymer even for very thin (approximately $2 \mathrm{~nm}$ thick in this study) layers. In the present study only the two more hydrophobic crosslinkers were able to react with all thiol groups within the hydrophobic polymer film. From the two hydrophobic crosslinkers that reacted, bismaleimidohexane (BMH) and $1,1^{\prime}$-(methylenedi-4,1-phenylene) bismaleimide (BMDPM), BMDPM produced much more robust films, as judged by stability of the films when exposed to temperatures of $80^{\circ} \mathrm{C}$. The primary difference between these two crosslinkers is the more flexible nature of $\mathrm{BMH}$, compared to the rather rigid ring-containing structure of BMDPM (Figure 1). A possible explanation for the effectiveness of BMDPM is that more 


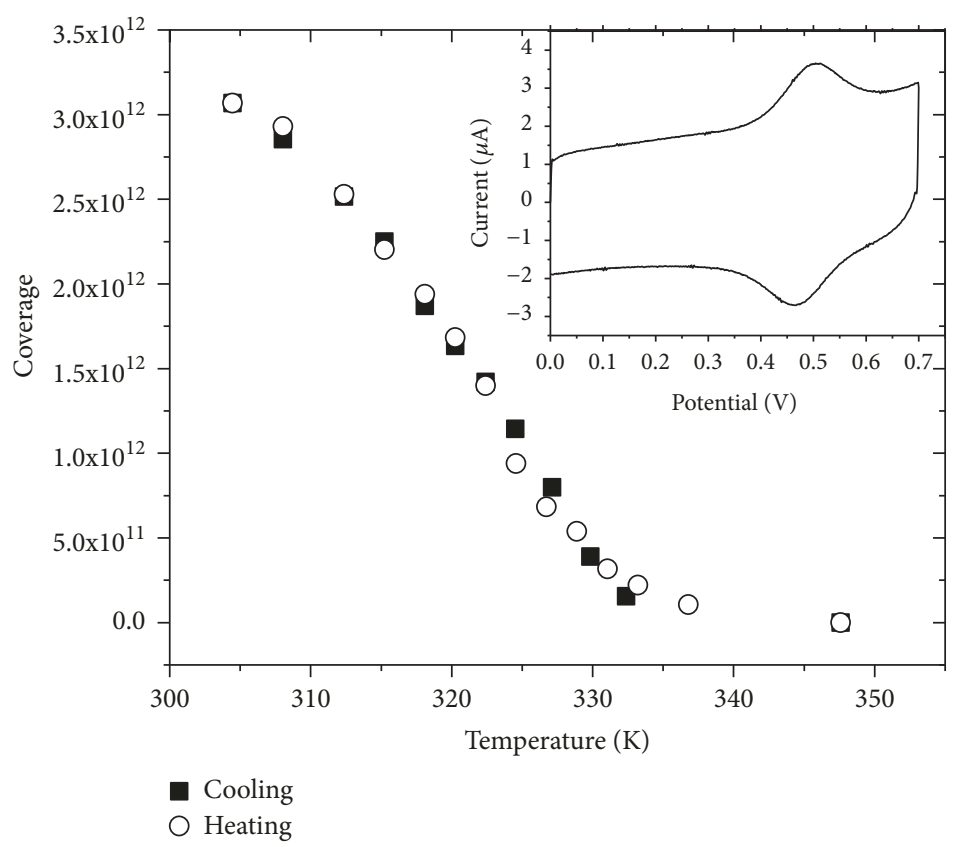

Figure 7: A sample melting curve measured on a DNA monolayer immobilized to a BMDPM-crosslinked PMPMS film. The probe sequence is $5^{\prime}$-ATG ATG ATG CAG TGT GAG TT-3', which was also used in the thermodynamic studies of reference [4]. Inserted: CV curve at $T=$ $305 \mathrm{~K}$.

rigid and hence extended crosslinkers are more likely to crosslink two different polymer chains as opposed to two locations on the same chain, what should better stabilize the layer. However, rather long times were required for crosslinks to fully form-up to 17 hrs-indicating that once a BMDPM crosslinker incorporated via one terminal maleimide, it can take hours for its other end to successfully react with a second thiol group. These long times may be required because some crosslinks are conformationally difficult to realize. Nevertheless, with the optimized crosslinking protocol, highly thermostable immobilization of biomolecules to solid supports is possible.

\section{Data Availability}

The data used to support the findings of this study are available from the corresponding author upon request.

\section{Conflicts of Interest}

The authors declare that there are no conflicts of interest regarding the publication of this paper.

\section{Acknowledgments}

This work was supported by the National Science Foundation [DMR 12-06754; CBET 16-00584] and by New York University.

\section{References}

[1] J. E. Forman, I. D. Walton, D. Stern, R. P. Rava, and M. O. Trulson, "Thermodynamics of Duplex Formation and Mismatch
Discrimination on Photolithographically Synthesized Oligonucleotide Arrays," in Molecular Modeling of Nucleic Acids, vol. 682 of ACS Symposium Series, pp. 206-228, American Chemical Society, Washington, DC, 1997.

[2] J. Xu and S. L. Craig, "Thermodynamics of DNA hybridization on gold nanoparticles," Journal of the American Chemical Society, vol. 127, no. 38, pp. 13227-13231, 2005.

[3] J. B. Fiche, A. Buhot, R. Calemczuk, and T. Livache, "Temperature effects on DNA chip experiments from surface plasmon resonance imaging: Isotherms and melting curves," Biophysical Journal, vol. 92, no. 3, pp. 935-946, 2007.

[4] H. Chiang and R. Levicky, "Effects of Chain-Chain Associations on Hybridization in DNA Brushes," Langmuir, vol. 32, no. 48, pp. 12603-12610, 2016.

[5] R. C. Welling and T. A. Knotts, "The effects of multiple probes on the hybridization of target DNA on surfaces," The Journal of Chemical Physics, vol. 142, no. 1, 2015.

[6] J. H. Watterson, P. A. E. Piunno, C. C. Wust, and U. J. Krull, "Effects of oligonucleotide immobilization density on selectivity of quantitative transduction of hybridization of immobilized DNA," Langmuir, vol. 16, no. 11, pp. 4984-4992, 2000.

[7] C. Chen, W. Wang, J. Ge, and X. S. Zhao, "Kinetics and thermodynamics of DNA hybridization on gold nanoparticles," Nucleic Acids Research, vol. 37, no. 11, pp. 3756-3765, 2009.

[8] T. J. Petty, C. E. Wagner, and A. Opdahl, "Influence of attachment strategy on the thermal stability of hybridized DNA on gold surfaces," Langmuir, vol. 30, no. 50, pp. 15277-15284, 2014.

[9] K. A. Peterlinz, R. M. Georgiadis, T. M. Herne, and M. J. Tarlov, "Observation of hybridization and dehybridization of thiol-tethered DNA using two-color surface plasmon resonance spectroscopy," Journal of the American Chemical Society, vol. 119, no. 14, pp. 3401-3402, 1997.

[10] P. A. E. Piunno, J. H. Watterson, C. C. Kotoris, and U. J. Krull, "Alteration of the selectivity of hybridization of immobilized 
oligonucleotide probes by co-immobilization with charged oligomers of ethylene glycol," Analytica Chimica Acta, vol. 534, no. 1, pp. 53-61, 2005.

[11] J. Fuchs, J.-B. Fiche, A. Buhot, R. Calemczuk, and T. Livache, "Salt concentration effects on equilibrium melting curves from DNA microarrays," Biophysical Journal, vol. 99, no. 6, pp. 18861895, 2010.

[12] S. L. Biswal, D. Raorane, A. Chaiken, H. Birecki, and A. Majumdar, "Nanomechanical detection of DNA melting on microcantilever surfaces," Analytical Chemistry, vol. 78, no. 20, pp. 71047109, 2006.

[13] H. Nasef, V. C. Ozalp, V. Beni, and C. K. O’Sullivan, "Melting temperature of surface-tethered DNA," Analytical Biochemistry, vol. 406, no. 1, pp. 34-40, 2010.

[14] W. Qiao, H. Chiang, H. Xie, and R. Levicky, "Surface vs. solution hybridization: Effects of salt, temperature, and probe type," Chemical Communications, vol. 51, no. 97, pp. 17245-17248, 2015.

[15] A. Vainrub, N. Deluge, X. Zhang, X. Zhou, and X. Gao, "Predicting DNA duplex stability on oligonucleotide arrays," Methods in Molecular Biology, vol. 382, pp. 393-403, 2007.

[16] M. Iwakura, D. Nakamura, T. Takenawa, and Y. Mitsuishi, "An approach for protein to be completely reversible to thermal denaturation even at autoclave temperatures," Protein Engineering, Design and Selection, vol. 14, no. 8, pp. 583-589, 2001.

[17] U. Bora, P. Sharma, S. Kumar, K. Kannan, and P. Nahar, "Photochemical activation of a polycarbonate surface for covalent immobilization of a protein ligand," Talanta, vol. 70, no. 3, pp. 624-629, 2006.

[18] C. Thapliyal, N. Jain, N. Rashid, and P. Chaudhuri Chattopadhyay, "Kinetics and thermodynamics of the thermal inactivation and chaperone assisted folding of zebrafish dihydrofolate reductase," Archives of Biochemistry and Biophysics, vol. 637, pp. 21-30, 2018.

[19] C. C. Lee, G. A. McMillin, N. Babic, R. Melis, and K.-T. J. Yeo, "Evaluation of a CYP2C19 genotype panel on the GenMark eSensor ${ }^{\circledR}$ platform and the comparison to the Autogenomics Infiniti $^{\mathrm{TM}}$ and Luminex CYP2C19 panels," Clinica Chimica Acta, vol. 412, no. 11-12, pp. 1133-1137, 2011.

[20] Z. Li, R. Jin, C. A. Mirkin, and R. L. Letsinger, "Multiple thiolanchor capped DNA-gold nanoparticle conjugates," Nucleic Acids Research, vol. 30, no. 7, pp. 1558-1562, 2002.

[21] N. Phares, R. J. White, and K. W. Plaxco, "Improving the stability and sensing of electrochemical biosensors by employing trithiol-anchoring groups in a six-carbon self-assembled monolayer," Analytical Chemistry, vol. 81, no. 3, pp. 1095-1100, 2009.

[22] J. A. Dougan, C. Karlsson, W. E. Smith, and D. Graham, "Enhanced oligonucleotide-nanoparticle conjugate stability using thioctic acid modified oligonucleotides," Nucleic Acids Research, vol. 35, no. 11, pp. 3668-3675, 2007.

[23] T. Sakata, S. Maruyama, A. Ueda, H. Otsuka, and Y. Miyahara, "Stable immobilization of an oligonucleotide probe on a gold substrate using tripodal thiol derivatives," Langmuir, vol. 23, no. 5, pp. 2269-2272, 2007.

[24] J. Sharma, R. Chhabra, H. Yan, and Y. Liu, "A facile in situ generation of dithiocarbamate ligands for stable gold nanoparticleoligonucleotide conjugates," Chemical Communications, no. 18, pp. 2140-2142, 2008.

[25] L. Civit, A. Fragoso, and C. K. O'Sullivan, “Thermal stability of diazonium derived and thiol-derived layers on gold for application in genosensors," Electrochemistry Communications, vol. 12, no. 8, pp. 1045-1048, 2010.
[26] T. Livache, A. Roget, E. Dejean, C. Barthet, G. Bidan, and R. Teoule, "Preparation of a DNA matrix via an electrqchemically directed copolymerization of pyrrole and oligonucleotides bearing a pyrrole group," Nucleic Acids Research, vol. 22, no. 15, pp. 2915-2921, 1994.

[27] P. A. Johnson and R. Levicky, "Polymercaptosiloxane Anchor Films for Robust Immobilization of Biomolecules to Gold Supports," Langmuir, vol. 19, no. 24, pp. 10288-10294, 2003.

[28] D. Ge, X. Wang, K. Williams, and R. Levicky, “Thermostable DNA immobilization and temperature effects on surface hybridization," Langmuir, vol. 28, no. 22, pp. 8446-8455, 2012.

[29] U. Oesch and J. Janata, "Electrochemical study of gold electrodes with anodic oxide films-I. Formation and reduction behaviour of anodic oxides on gold," Electrochimica Acta, vol. 28, no. 9, pp. 1237-1246, 1983.

[30] G. Shen, N. Tercero, M. A. Gaspard, B. Varughese, K. Shepard, and R. Levicky, "Charging behavior of single-stranded DNA polyelectrolyte brushes," Journal of the American Chemical Society, vol. 128, no. 26, pp. 8427-8433, 2006.

[31] W. Scheider, "Theory of the frequency dispersion of electrode polarization. Topology of networks with fractional power frequency dependence," The Journal of Physical Chemistry C, vol. 79, no. 2, pp. 127-136, 1975.

[32] G. J. Brug, A. L. G. Van den Eeden, M. Sluyters-Rehbach, and J. H. Sluyters, "The analysis of electrode impedances complicated by the presence of a constant phase element," Journal of Electroanalytical Chemistry and Interfacial Electrochemistry, vol. 176, no. 1-2, pp. 275-295, 1984.

[33] P. A. Johnson and R. Levicky, "X-ray photoelectron spectroscopy and differential capacitance study of thiol-functional polysiloxane films on gold supports," Langmuir, vol. 20, no. 22, pp. 9621-9627, 2004.

[34] S. Pilehvar, T. Dierckx, R. Blust, T. Breugelmans, and K. De Wael, "An electrochemical impedimetric aptasensing platform for sensitive and selective detection of small molecules such as chloramphenicol," Sensors, vol. 14, no. 7, pp. 12059-12069, 2014.

[35] D. Ge and R. Levicky, "A comparison of five bioconjugatable ferrocenes for labeling of biomolecules," Chemical Communications, vol. 46, no. 38, pp. 7190-7192, 2010.

[36] N. Tercero, K. Wang, P. Gong, and R. Levicky, "Morpholino monolayers: Preparation and label-free DNA analysis by surface hybridization," Journal of the American Chemical Society, vol. 131, no. 13, pp. 4953-4961, 2009.

[37] Y. Rogers, P. Jiang-Baucom, Z.-J. Huang, V. Bogdanov, S. Anderson, and M. T. Boyce-Jacino, "Immobilization of oligonucleotides onto a glass support via disulfide bonds: A method for preparation of DNA microarrays," Analytical Biochemistry, vol. 266, no. 1, pp. 23-30, 1999.

[38] N. R. Markham and M. Zuker, "UNAFold: Software for nucleic acid folding and hybridization," Methods in Molecular Biology, vol. 453, pp. 3-31, 2008.

[39] Y. Okahata, M. Kawase, K. Niikura, F. Ohtake, H. Furusawa, and Y. Ebara, "Kinetic measurements of DNA hybridization on an oligonucleotide-immobilized 27-MHz quartz crystal microbalance," Analytical Chemistry, vol. 70, no. 7, pp. 1288-1296, 1998. 

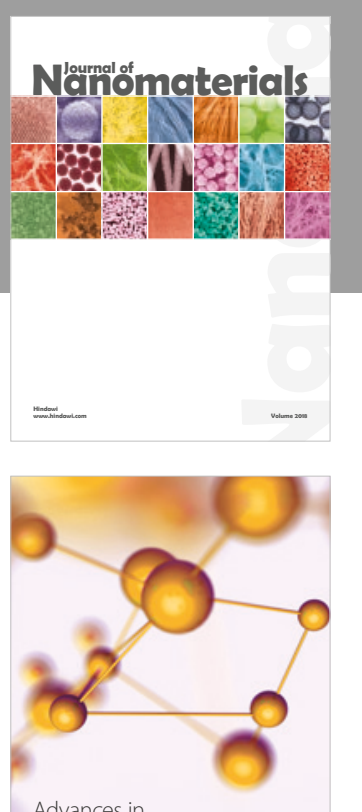

Physical Chemistry
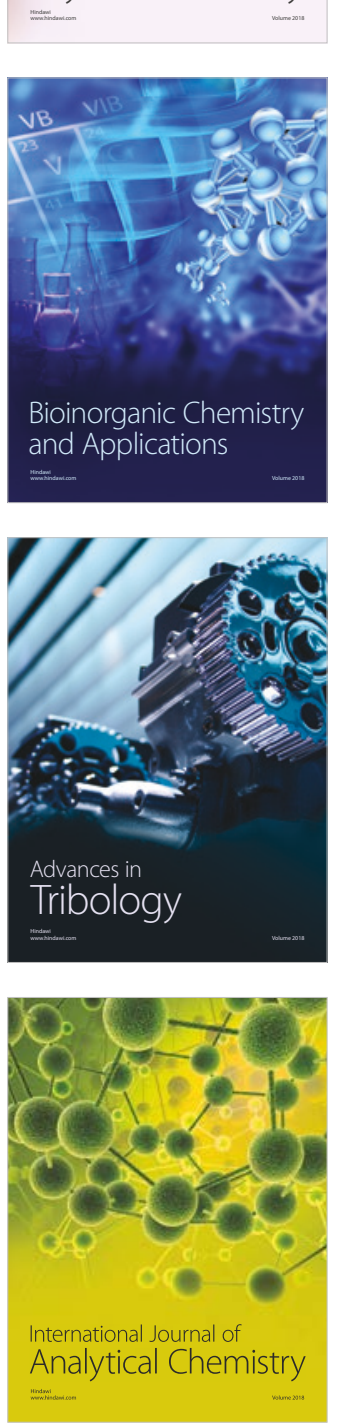

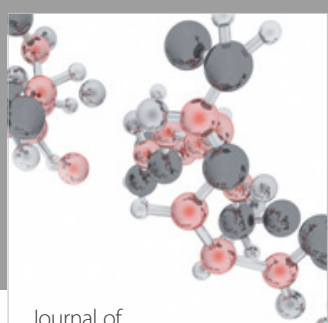

Analytical Methods

in Chemistry

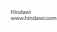

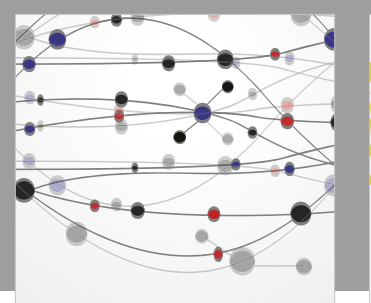

The Scientific World Journal

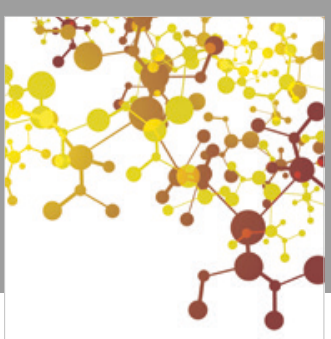

Journal of

Applied Chemistry
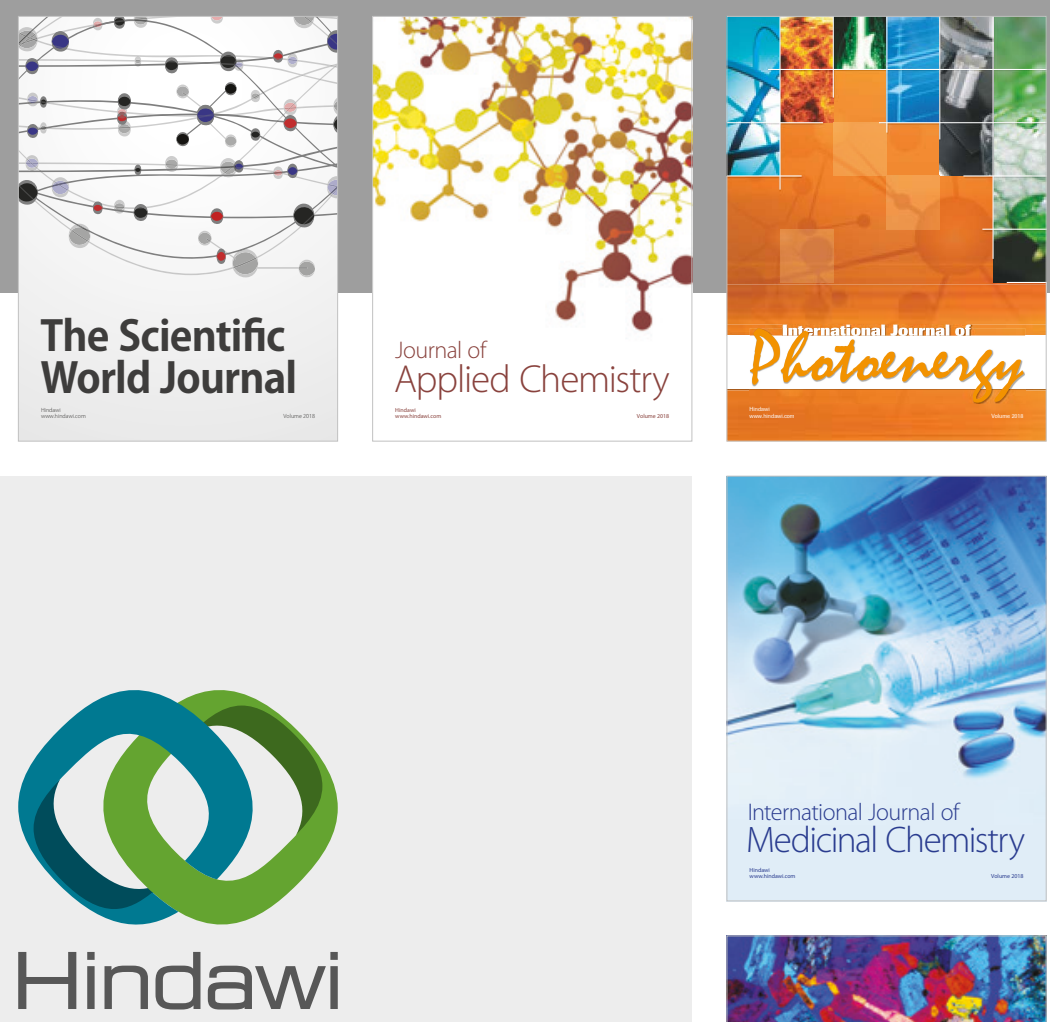

Submit your manuscripts at

www.hindawi.com
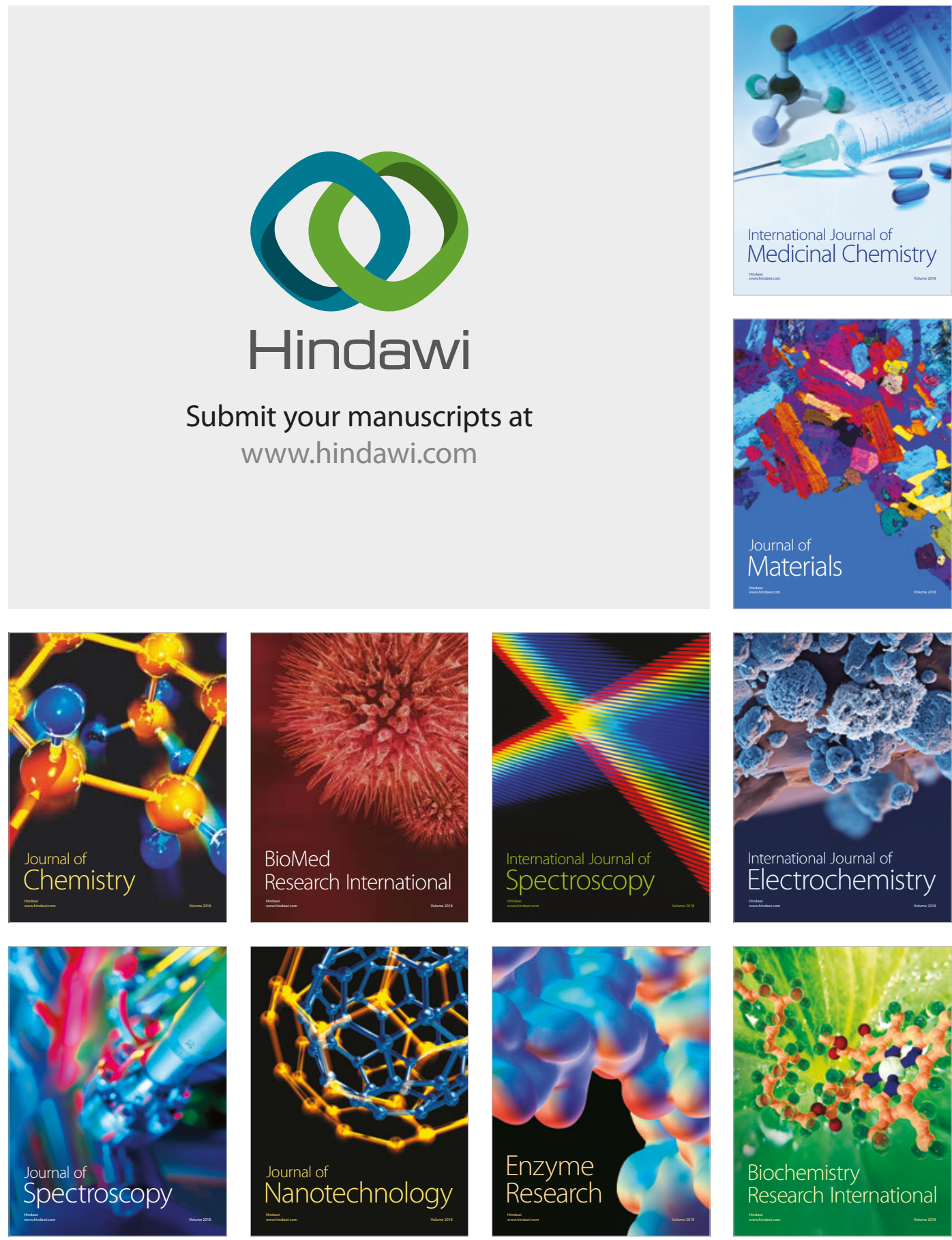
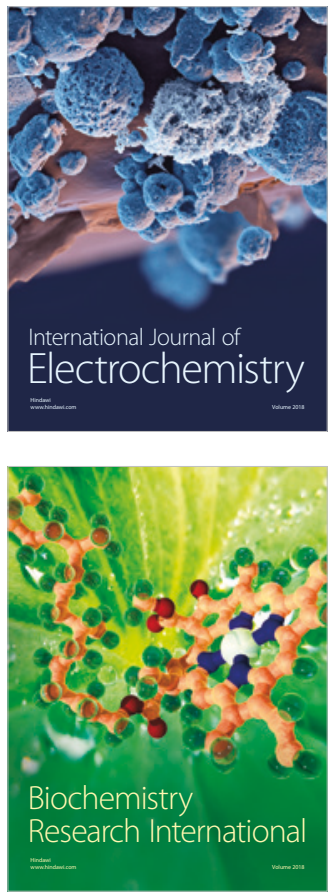